\title{
Tree nut snack consumption is associated with better diet quality and CVD risk in the UK adult population: National Diet and Nutrition Survey (NDNS) 2008-2014
}

\author{
Vita Dikariyanto*, Sarah E Berry, Gerda K Pot, Lucy Francis, Leanne Smith and \\ Wendy L Hall* \\ Department of Nutritional Sciences, School of Life Course Sciences, Faculty of Life Sciences and Medicine, King's \\ College London, London SE 1 9NH, UK
}

Submitted 13 July 2018: Final revision received 15 August 2019: Accepted 9 September 2019: First published online 28 February 2020

\begin{abstract}
Objectives: To examine associations of tree nut snack (TNS) consumption with diet quality and cardiovascular disease (CVD) risk in UK adults from National Diet and Nutrition Survey (NDNS) 2008-2014.

Design: Cross-sectional analysis using data from 4-d food diaries, blood samples and physical measurements for CVD risk markers. To estimate diet quality, modified Mediterranean Diet Score (MDS) and modified Healthy Diet Score (HDS) were applied. Associations of TNS consumption with diet quality and markers of CVD risk were investigated using survey-adjusted multivariable linear regression adjusted for sex, age, ethnicity, socio-economic and smoking status, region of residency and total energy and alcohol intake.

Setting: UK free-living population.

Subjects: 4738 adults ( $\geq 19$ years).

Results: TNS consumers had higher modified MDS and HDS relative to nonconsumers. TNS consumers also had lower BMI, WC, SBP and DBP and higher HDL compared to non-consumers, although a dose-related fully adjusted significant association between increasing nut intake (g per $4184 \mathrm{~kJ} / 1000 \mathrm{kcal}$ energy intake) and lower marker of CVD risk was only observed for SBP. TNS consumption was also associated with higher intake of total fat, mono-, $n-3$ and $n-6$ polyunsaturated fatty acids, fibre, vitamin A, thiamin, folate, vitamin C, vitamin E, potassium, magnesium, phosphorus, selenium and iron; and lower intake of saturated fatty acids, trans fatty acids, total carbohydrate, starch, free sugar, sodium and chloride. Conclusions: TNS consumers report better dietary quality and consumption was associated with lower CVD risk factors. Encouraging replacement of less healthy snacks with TNS should be encouraged as part of general dietary guidelines.
\end{abstract}

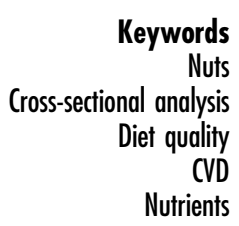

An average of 2.55 snacks per day are consumed in the UK and Ireland, with over a third of these snacks being confectionary or crisps/popcorn/nuts ${ }^{(1)}$. Nuts are a popular snack as shown by the growing trend for consuming tree nuts over the past 10 years $^{(2)}$. North America was the region with the highest production; however, it was Europe that was the largest consumer in the world. Almonds (Prunusdulcis), walnuts (Juglansregia), pecans (Caryaillinoinensis), pine nuts (Pinuspinea), cashews (Anacardiumoccidentale), macadamia nuts (Macadamia), hazelnuts (Corylusavelana), pistachios (Pistaciavera), Brazil nuts (Bertholletiaexcelsa) and chestnuts (Castanea) are examples of edible tree nuts that are produced commercially ${ }^{(2)}$.
Almonds, walnuts, pecans, pine nuts, cashews, macadamia nuts, hazelnuts, pistachios and Brazil nuts differ to some extent in their nutrient profiles. However, tree nuts are generally energy-dense, with a high proportion of fat made up of unsaturated fatty acids; low in sodium; and rich in plant-based protein, dietary fibre, and micronutrients, including niacin, vitamin $\mathrm{B}_{6}$, vitamin $\mathrm{E}$, vitamin $\mathrm{K}$, folic acid, calcium, magnesium, potassium, selenium, phosphorus and zinc. Tree nuts are also rich in phytosterols and (poly)phenols, which promote antioxidant and antiinflammatory pathways ${ }^{(3-5)}$. Because of these properties, tree nuts and health outcomes have been the focus of many human clinical trials and observational studies. 
Previous dietary intervention studies showed that tree nut consumption resulted in lowered type- 2 diabetes and cardiovascular disease (CVD) risk factors. Walnut, almond, pistachio, macadamia nut, cashew and hazelnut consumption favourably modified blood lipid profile ${ }^{(6-15)}$, mixed nuts improved insulin sensitivity ${ }^{(14)}$, and walnuts lowered inflammatory markers ${ }^{(16)}$ and improved endotheliumdependent vasodilation ${ }^{(17)}$, all of which would be predicted to reduce the risk of CVD. Furthermore, contrary to popular perception, nut-enriched diets are not linked with increased risk of weight gain ${ }^{(18)}$ and tree nut consumption has been shown to assist weight loss as part of an energy-restricted diet in obese or overweight subjects $^{(19)}$

Cross-sectional analysis of tree nut consumption and indicators of diet quality and cardiovascular health have also been undertaken. In the US adult population ( $\geq 19$ years), using the National Health and Nutrition Examination Survey (NHANES) 2005-2010 database ( $n$ 14 386) based on 24-h dietary recalls, it was reported that tree nut consumption was linked to lower BMI, waist circumference (WC), systolic blood pressure (SBP), and insulin resistance index (HOMA-IR) and higher highdensity lipoprotein (HDL-C) adjusted for age, sex, ethnicity, poverty index ratio, physical activity level, smoking status and alcohol intake ${ }^{(20)}$. O'Neil et al. (2015) also showed that tree nut consumers, compared to non-consumers, had significantly higher diet quality scores (HEI-2005, a diet quality score widely used in the USA) and greater nutrient adequacy for dietary fibre, vitamin A, vitamin $\mathrm{E}$, vitamin $\mathrm{C}$, folate, calcium, iron, magnesium, zinc and potassium ${ }^{(21)}$.

The purpose of the current study was to examine associations between tree nut snack (TNS) consumption and diet quality, and CVD risk markers, in a nationally representative UK adult population, using data from the UK National Diet and Nutrition Survey (NDNS) rolling programme 2008-2014. Dietary data were derived from estimated 4-d food diaries in a population of 4738 adults $(\geq 19 \text { years })^{(22,23)}$, which differs from the NHANES analysis 2005-2010, which was based on two multiple pass 24-h dietary recalls in a larger population of 14386 adults $^{(21)}$. The hypothesis of the current study was that greater TNS consumption would be associated with higher diet quality, greater nutrient adequacy, and lower prevalence of CVD risk markers in UK adults.

\section{Materials and Methods}

\section{The National Diet and Nutrition Survey Rolling} Programme (NDNS-RP) and study population

The NDNS-RP is a long-running government-funded scheme to assess diet, nutrient intake and nutritional status of the general population ( $>1.5$ years) living in private households in the UK (England, Scotland, Wales and North Ireland) ${ }^{(22,23)}$. Random sampling was carried out on addresses throughout the UK. A single address could have multiple households and a household in an address was selected randomly. An adult in the household was also randomly selected. Selected participants were requested to complete a 4-d estimated food diary, interviewed to collect information, such as dietary habits, socio-demographic background and lifestyle as well as anthropometrically measured and blood sample taken ${ }^{(22,23)}$.

The survey involves two stages: (i) interview visits to collect information on socio-demography, administer the 4-d food diaries, and carry out anthropometric measurements, and (ii) a nurse visit to do further physical measurements and collect blood and 24-h urine samples ${ }^{(22,23)}$. Following venepuncture, an EDTA and a serum gel monovette tube from each participant's sample set were sent by post, to the Immunology and Biochemistry Laboratory at Addenbrooke's Hospital in Cambridge for prompt analysis. The remaining samples (lithium heparin, serum or fluoride blood monovette tubes) were processed and stored below $-40^{\circ} \mathrm{C}$ (or at a maximum of $-20^{\circ} \mathrm{C}$ where $-40^{\circ} \mathrm{C}$ facilities were not available), before being transported on dry ice to the Human Nutrition Research (HNR) facility for analysis. The cross-sectional analysis reported here included data from adult participants ( $\geq 19$ years, $n$ 4738), who completed a 4-d estimated food diary in the NDNS-RP 2008-2014 $(\text { years } 1-6)^{(22,23)}$.

\section{CVD risk markers}

Body mass index (BMI; $\mathrm{kg} / \mathrm{m}^{2}$ ), waist circumference (WC; $\mathrm{cm}$ ), systolic blood pressure ( $\mathrm{SBP} ; \mathrm{mmHg}$ ), diastolic blood pressure (DBP; $\mathrm{mmHg}$ ), total cholesterol (TC; mmol/l), triglycerides (TAG; mmol/l), HDL-cholesterol (HDL-C; mmol/l), LDL-cholesterol (LDL-C; mmol/l), TC:HDL-C (the ratio of TC and HDL-C) and C-reactive protein (CRP; $\mathrm{mg} / \mathrm{l})$ were CVD risk markers included in the analysis. Interviewer measurement protocols and procedures for blood sample collection, processing, analysis and quality controls are detailed elsewhere ${ }^{(22,23)}$. Body height and weight were measured using a portable stadiometer and a weight scale, and BMI was calculated by fieldworkers. WC measurement was taken using a tape measure. The discrepancy tolerances of repeat measurement readings were not detailed in the NDNS method protocols. Omron HEM907, an automated validated monitor, was used to measure blood pressure in a sitting position after a five-minute rest. Trained fieldworkers took blood pressure measurements three times and results were presented based on the mean value of second and third readings with one-minute intervals ${ }^{(22,23)}$.

\section{Diet quality indices}

To estimate diet quality, two existing diet scores were used: the Mediterranean Diet Score (MDS) ${ }^{(24)}$ and Healthy Diet Score (HDS) ${ }^{(25)}$. Maynard et al. (2004) developed HDS based on Healthy Diet Indicator (HDI) and the UK 
guidelines at that point in time, as recommended by the Committee on Medical Aspects of Food Policy (COMA) ${ }^{(25)}$. Modifications were applied to HDS for the current study to reflect UK current recommendations ${ }^{(22,26-30)}$, and nuts were removed from the MDS scoring system as appropriate for the current study on diet and health associations with nut consumption. The potential top score of the modified MDS remained the same: 9, but the modified HDS had a potential top score of 14 while the original HDS scoring range was 0-12 (see Table A2 in Appendices). Tables A1 and A2 in Appendices show original and modified items of MDS and HDS items, respectively.

\section{Statistical analysis}

Prior to statistical analysis, TNS intake was defined and determined. TNS consumption was defined as: (i) any amount of consumption or (ii) $\geq 7.08 \mathrm{~g}(1 / 4 \mathrm{Oz})$ of TNS. The $\geq 7.08 \mathrm{~g}(1 / 4 \mathrm{Oz})$ cut-off was adopted to facilitate comparisons with previous cross-sectional analysis of associations between tree nut consumption and dietary scores/ nutrient adequacy in a US adult population ${ }^{(21)}$. Data on TNS consumption were isolated from the database prior to statistical analysis and total TNS intake was calculated. Tree nuts included were almonds, walnuts, pecans, pine nuts, cashews, macadamia nuts, hazelnuts, pistachios, Brazil nuts and chestnuts. Although the US Food and Drug Administration recognises coconuts as a tree nut, they were excluded since they are fruits of palm trees and not commonly consumed whole as a snack food. Peanuts were also excluded since they are classified as legumes.

Statistical analysis was carried out using SPSS IBM 23, and a two-sided $P$-value of 0.05 was considered statistically significant. Data are presented as adjusted means (95\% CI) for individual nutrient intakes, total diet quality scores as well as levels of CVD risk markers, and as medians (with IQRs) for the amount of TNS consumed and age. To examine whether there was a statistically significant association between tree nut consumption and alcohol and total energy intakes as well as demographic variables, that is, age, sex, ethnicity, socio-economic and smoking status and region of residency, survey-adjusted generalised linear model (GLM) with a binary logistic link function was used. Survey-adjusted GLM with a linear link function (predictors: age, sex, ethnicity, socio-economic and smoking status, region of residency, total energy and alcohol intake) was used to examine whether there were significant differences between TNS consumers and non-consumers in their diet quality scores, nutrient intakes and CVD risk markers. To investigate dose-response associations between TNS consumption ( $\mathrm{g} / 4184 \mathrm{~kJ}$ energy intake) and diet quality and CVD risk markers, survey-adjusted multivariable linear regression models were used adjusting for the same covariates mentioned above. Normal residual distributions were checked by visual inspection of histogram and Q-Q plots; data with non-normally distributed residuals were log transformed using $\log _{10}$ for analysis of survey-adjusted GLM and multivariable linear regression. The results of analysis were back transformed into the geometric mean values. Homoscedasticity was checked by plotting the standardised residuals of dependent variables and predictors.

During the analysis, the weight factor provided by the NDNS database resource was applied to adjust for nonresponse and known socio-economic differences in the survey to ensure that the data were nationally representative for the UK population and reducing selection bias and non-response bias ${ }^{(31,32)}$. The weight factor used is wti_Y14 (weight for individual and diary-all ages, combined Y1-4) and wti_Y56 (weight for individual and diary-all ages, combined Y5-6) for investigating differences in diet quality scores and nutrient intakes between TNS consumers and non-consumers, associations between tree nut consumption and demographic variables and multivariable linear regression including diet quality scores. Weight factors wtn_Y14 (weight for nurse-all ages, combined Y1-4) and wtn_Y56 (weight for nurse-all ages, combined Y5-6) were used for GLM and multivariable linear regression including variables BMI, WC and blood pressure; and wtb_Y14 (weight for blood-all ages, combined Y1-4) and wtb_Y56 (weight for blood-all ages, combined Y5-6) were used for GLM and multivariable linear regression for blood analyte variables including CRP and lipids ${ }^{(31,32)}$.

\section{Results}

\section{Demographic information}

Table 1 shows background characteristics of TNS consumers and non-consumers. Median TNS-A (any amount of TNS intake) consumption ( $n$ 484) contributed $0.8 \%$ of total energy intake while median consumption in the TNS-B group (including individuals who consumed $\geq 7 \cdot 08 \mathrm{~g}$ TNS per day, equivalent to $1 / 4 \mathrm{Oz}, n 224$ ) was $2.3 \%$ of total energy intake. On average, TNS consumers were significantly older than non-consumers and were more likely to be female and non-smokers. TNS-A consumption was significantly associated with the demographic factors included, such as sex, ethnicity, socio-economic status, smoking status and region of residency. TNS-B consumption was also significantly associated with these demographic variables, except region of residency.

\section{Diet quality scores}

Geometric estimated marginal mean total scores of modified MDS were significantly higher in TNS-A consumers (5.9; $95 \%$ CI 5.2, 6.6) compared with non-consumers (4.9; $95 \%$ CI 4.4, 5.4; P<0.001). Similarly, geometric estimated marginal mean total scores for the modified HDS were significantly higher in TNS-A consumers $(6 \cdot 1 ; 95 \%$ CI $5 \cdot 5,6 \cdot 8)$ compared with non-consumers $(5 \cdot 4 ; 95 \% \mathrm{CI}$ 4.9, 6.0; $P<0.001)$. Results for TNS-B consumers were 
Table 1 Background characteristics of tree nut snack (TNS) consumers compared to non-consumers in the UK adult population ( $\geq 19$ years) based on NDNS 2008-2014, $n 4738$

\begin{tabular}{|c|c|c|c|c|c|c|c|}
\hline & & \multicolumn{3}{|c|}{ TNS-A } & \multicolumn{3}{|c|}{ TNS-B } \\
\hline & & $\begin{array}{l}\text { Consumers, } \\
\text { n } 484\end{array}$ & $\begin{array}{c}\text { Non-consumers, } \\
n 4254\end{array}$ & $P$-value & $\begin{array}{l}\text { Consumers, } \\
n 224\end{array}$ & $\begin{array}{l}\text { Non-consumers, } \\
n 4514\end{array}$ & $P$-value \\
\hline \multirow{6}{*}{$\begin{array}{l}\text { Amount of tree nuts } \\
\text { consumed }\end{array}$} & Gram & & & & & & \\
\hline & Median & 6.5 & & & $14 \cdot 0$ & & \\
\hline & IQR & $10 \cdot 8$ & & & $10 \cdot 6$ & & \\
\hline & $\%$ Total energy intake & & & & & & \\
\hline & Median & $0 \cdot 8$ & & & $2 \cdot 3$ & & \\
\hline & IQR & $2 \cdot 2$ & & & $5 \cdot 1$ & & \\
\hline \multicolumn{2}{|l|}{ Age } & & & $<0.001^{*}$ & & & $<0.001^{*}$ \\
\hline Median & & 51 & 48 & & 53 & 48 & \\
\hline IQR & & 24 & 27 & & 24 & 27 & \\
\hline \multirow[t]{2}{*}{$\operatorname{Sex}(\%)$} & Male & $31 \cdot 1$ & $41 \cdot 6$ & $<0.001^{*}$ & $32 \cdot 8$ & $40 \cdot 8$ & $<0.001^{*}$ \\
\hline & Female & 68.9 & $58 \cdot 4$ & & $67 \cdot 2$ & $59 \cdot 2$ & \\
\hline \multirow[t]{5}{*}{ Ethnicity (\%) } & White & $87 \cdot 8$ & $93 \cdot 8$ & $0.003^{*}$ & $88 \cdot 9$ & 93.4 & $0.016^{*}$ \\
\hline & Mixed ethnic group & $1 \cdot 7$ & 0.9 & & 0.9 & 1.0 & \\
\hline & Black or Black British & $1 \cdot 6$ & $2 \cdot 0$ & & $1 \cdot 7$ & 1.9 & \\
\hline & Asian or Asian British & $5 \cdot 9$ & $2 \cdot 2$ & & $5 \cdot 4$ & $2 \cdot 4$ & \\
\hline & Any other group & $3 \cdot 1$ & $1 \cdot 2$ & & 3.0 & $1 \cdot 3$ & \\
\hline \multirow[t]{4}{*}{ Region (\%) } & England & $68 \cdot 1$ & $54 \cdot 8$ & $0.003^{*}$ & $64 \cdot 8$ & $55 \cdot 8$ & $0 \cdot 131$ \\
\hline & Scotland & $11 \cdot 9$ & $17 \cdot 9$ & & 11.9 & $17 \cdot 5$ & \\
\hline & Wales & $12 \cdot 5$ & $14 \cdot 8$ & & $16 \cdot 7$ & 14.4 & \\
\hline & Northern Ireland & $7 \cdot 5$ & $12 \cdot 6$ & & $6 \cdot 6$ & $12 \cdot 3$ & \\
\hline \multirow[t]{9}{*}{$\begin{array}{l}\text { Socio-economic } \\
\text { status (\%) }\end{array}$} & $\begin{array}{l}\text { Higher managerial and } \\
\text { professional occupations }\end{array}$ & $27 \cdot 2$ & $13 \cdot 7$ & $<0.001^{*}$ & $25 \cdot 9$ & $14 \cdot 6$ & $<0.001^{*}$ \\
\hline & $\begin{array}{l}\text { Lower managerial and } \\
\text { professional occupations }\end{array}$ & $31 \cdot 2$ & $23 \cdot 1$ & & $26 \cdot 4$ & $23 \cdot 9$ & \\
\hline & Intermediate occupations & 8.2 & $10 \cdot 5$ & & $9 \cdot 7$ & $10 \cdot 3$ & \\
\hline & $\begin{array}{l}\text { Small employers and own } \\
\text { account workers }\end{array}$ & $11 \cdot 0$ & $10 \cdot 4$ & & $11 \cdot 1$ & $10 \cdot 4$ & \\
\hline & $\begin{array}{l}\text { Lower supervisory and } \\
\text { technical occupations }\end{array}$ & $6 \cdot 5$ & $9 \cdot 5$ & & $7 \cdot 0$ & $9 \cdot 3$ & \\
\hline & Semi-routine occupations & $9 \cdot 4$ & $15 \cdot 1$ & & $13 \cdot 2$ & $14 \cdot 6$ & \\
\hline & Routine occupations & 3.4 & $12 \cdot 9$ & & 4.5 & $12 \cdot 2$ & \\
\hline & Never worked & $1 \cdot 3$ & $3 \cdot 1$ & & 0.7 & $3 \cdot 0$ & \\
\hline & Others & 1.9 & $1 \cdot 7$ & & 1.5 & $1 \cdot 8$ & \\
\hline \multirow[t]{3}{*}{ Smoking status (\%) } & Current smoker & $11 \cdot 7$ & $25 \cdot 0$ & $<0.001^{*}$ & $12 \cdot 5$ & $24 \cdot 2$ & $<0.001^{\star}$ \\
\hline & Ex-Regular smoker & $25 \cdot 7$ & $23 \cdot 6$ & & $27 \cdot 3$ & $23 \cdot 7$ & \\
\hline & Never regular smoker & $62 \cdot 6$ & 51.4 & & $60 \cdot 2$ & $52 \cdot 2$ & \\
\hline \multicolumn{2}{|l|}{ Alcohol intake (g/d) } & & & $0.012^{*}$ & & & $0.002^{*}$ \\
\hline \multirow{2}{*}{\multicolumn{2}{|c|}{$\begin{array}{l}\text { Median } \\
\text { IQR }\end{array}$}} & $6 \cdot 0$ & 0.7 & & 4.4 & $1 \cdot 8$ & \\
\hline & & 18.4 & $16 \cdot 7$ & & $17 \cdot 0$ & $16 \cdot 9$ & \\
\hline \multicolumn{2}{|l|}{ Energy intake (kJ/d) } & & & $<0.001^{*}$ & & & $<0.001^{*}$ \\
\hline \multirow{2}{*}{\multicolumn{2}{|c|}{$\begin{array}{l}\text { Unadjusted mean } \\
\text { SD }\end{array}$}} & $7950 \cdot 9$ & $7325 \cdot 3$ & & $8168 \cdot 8$ & $7350 \cdot 5$ & \\
\hline & & $2023 \cdot 8$ & 2364.4 & & $2087 \cdot 0$ & $2344 \cdot 7$ & \\
\hline
\end{tabular}

This is a descriptive table. Survey-adjusted generalised linear model with a linear binary logistic function was used to investigate the association between TNS consumption and demographic variables.

${ }^{\star} P$ was $<0.05$ indicating a significant association.

almost identical (data not shown). To investigate doseresponse associations between every gram increase in TNS consumption per $4184 \mathrm{~kJ}$ of adult's energy intake and diet quality scores, the survey-adjusted regression model was adjusted for age, sex, ethnicity, socio-economic and smoking status, alcohol and energy intakes. There was no dose response observed in the scores of modified MDS and modified HDS ( $P=0.726$ and $P=0.971$, respectively).

\section{Nutrient intake}

TNS consumers had significantly higher total energy, food energy, fat, cis-monounsaturated fatty acids, cis $n$ - 6 fatty acids, cis $n$-3 fatty acids (TNS-A only), intrinsic milk sugars and fibre intakes, as shown in Table 2. Saturated fatty acids, trans-fatty acids, total carbohydrate, starch, nonmilk extrinsic sugars, intrinsic milk sugar and starch and alcohol (TNS-B only) intakes were significantly lower in TNS consumers. For micronutrients, as shown in Table 2, fully adjusted analysis revealed that TNS consumers, relative to non-consumers, had significantly higher intakes of vitamin A (TNS-A only), vitamin E, thiamin, riboflavin (TNS-B only), folate, pantothenic acid, biotin, vitamin C, potassium, magnesium, phosphorus, iron, copper, zinc, manganese and selenium and lower intakes of sodium and chloride. However, there were no differences between groups for vitamins $\mathrm{D}$, riboflavin (TNS-A only), niacin equivalents, vitamin $\mathrm{B}_{6}$, vitamin $\mathrm{B}_{12}$, calcium and iodine. 


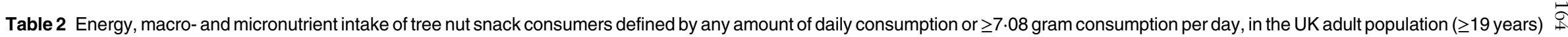
based on National Diet and Nutrition Survey 2008-2014, n 4738

\begin{tabular}{|c|c|c|c|c|c|c|c|c|c|c|}
\hline \multirow[b]{4}{*}{ Nutrients } & \multicolumn{4}{|c|}{ TNS-A } & \multirow[b]{4}{*}{$P$-value } & \multicolumn{4}{|c|}{ TNS-B } & \multirow[b]{4}{*}{$P$-value } \\
\hline & \multicolumn{2}{|c|}{ Consumers } & \multicolumn{2}{|c|}{ Non-consumers } & & \multicolumn{2}{|c|}{ Consumers } & \multicolumn{2}{|c|}{ Non-consumers } & \\
\hline & \multicolumn{2}{|r|}{$n 484$} & \multicolumn{2}{|c|}{$n 4254$} & & \multicolumn{2}{|c|}{$n 224$} & \multicolumn{2}{|c|}{$n 4514$} & \\
\hline & $\begin{array}{c}\text { Estimated } \\
\text { marginal } \\
\text { mean }\end{array}$ & $95 \% \mathrm{Cl}$ & $\begin{array}{c}\text { Estimated } \\
\text { marginal } \\
\text { mean }\end{array}$ & $95 \% \mathrm{Cl}$ & & $\begin{array}{c}\text { Estimated } \\
\text { marginal } \\
\text { mean }\end{array}$ & $95 \% \mathrm{Cl}$ & $\begin{array}{c}\text { Estimated } \\
\text { marginal } \\
\text { mean }\end{array}$ & $95 \% \mathrm{Cl}$ & \\
\hline \multicolumn{11}{|l|}{ Macronutrients (diet only, $\%$ food energy) ${ }^{*}$} \\
\hline Total energy (kJ) & $7365 \cdot 1 \dagger$ & $6335.8,8393.9$ & $6548 \cdot 0$ & $5532 \cdot 5,7563 \cdot 0$ & $<0.001$ & $7628 \cdot 3 \dagger$ & $6581.4,8675.5$ & $6782 \cdot 7$ & $5771 \cdot 8,7793.5$ & $<0.001$ \\
\hline Food energy $(\mathrm{kJ})$ & $7127.0 \dagger$ & $6155 \cdot 9,8098 \cdot 6$ & 6373.9 & $5415 \cdot 4,7332 \cdot 0$ & $<0.001$ & $7417 \cdot 8 \dagger$ & $6430 \cdot 0,8405 \cdot 2$ & $6580 \cdot 2$ & $5626 \cdot 6,7533 \cdot 3$ & $<0.001$ \\
\hline Protein & 17.4 & $15 \cdot 5,19 \cdot 2$ & $17 \cdot 3$ & $15 \cdot 5,19 \cdot 2$ & 0.827 & 17.5 & $15 \cdot 7,19 \cdot 4$ & $17 \cdot 3$ & $15 \cdot 5,19 \cdot 1$ & 0.384 \\
\hline Fat & $37.1 \dagger$ & $34 \cdot 2,40 \cdot 0$ & $35 \cdot 1$ & $32 \cdot 2,38.0$ & $<0.001$ & $37.6 \dagger$ & $34.7,40.5$ & $35 \cdot 2$ & $32 \cdot 3,38 \cdot 0$ & $<0.001$ \\
\hline Saturated fatty acids & $12 \cdot 2 \dagger$ & $10 \cdot 6,13 \cdot 7$ & 12.5 & $11.0,14.0$ & 0.035 & $11.8 \dagger$ & $10 \cdot 2,13 \cdot 3$ & $12 \cdot 3$ & $10 \cdot 8,13.9$ & 0.008 \\
\hline cis-Monounsaturated fatty acids & $14.3 \dagger$ & $13 \cdot 0,15 \cdot 6$ & $13 \cdot 0$ & $11 \cdot 8,14.3$ & $<0.001$ & $15 \cdot 0 \dagger$ & $13 \cdot 6,16 \cdot 3$ & $13 \cdot 1$ & $11.8,14.4$ & $<0.001$ \\
\hline cis $n-6$ fatty acids & $6 \cdot 2 \dagger$ & $5 \cdot 5,6 \cdot 9$ & $5 \cdot 3$ & $4 \cdot 6,6 \cdot 0$ & $<0.001$ & $6.6 \dagger$ & $5 \cdot 8,7 \cdot 3$ & 5.4 & $4 \cdot 7,6 \cdot 1$ & $<0.001$ \\
\hline cis $n-3$ fatty acids $\ddagger$ & $1.1 \dagger$ & $0.9,1.3$ & 1.0 & $0.8,1 \cdot 2$ & $<0.001$ & 1.0 & $0.8,1 \cdot 1$ & 1.0 & $0.9,1.1$ & 0.469 \\
\hline Trans fatty acids & $0.5 \dagger$ & $0.4,0.7$ & 0.6 & $0.5,0.7$ & $<0.001$ & $0.5 \dagger$ & $0.3,0.6$ & 0.6 & $0.4,0.7$ & $<0.001$ \\
\hline Carbohydrate & $45 \cdot 6 \dagger$ & $42 \cdot 4,48 \cdot 8$ & $47 \cdot 6$ & $44 \cdot 4,50 \cdot 8$ & $<0.001$ & $44.9 \dagger$ & $41 \cdot 7,48 \cdot 2$ & $47 \cdot 6$ & $44 \cdot 4,50 \cdot 7$ & $<0.001$ \\
\hline Total sugars & $17 \cdot 7$ & $14.5,20 \cdot 9$ & $17 \cdot 2$ & $14 \cdot 1,20 \cdot 4$ & 0.139 & $18 \cdot 1$ & $14.9,21.4$ & $17 \cdot 8$ & $14 \cdot 6,20 \cdot 9$ & 0.399 \\
\hline Starch & $27.9 \dagger$ & $25 \cdot 1,30 \cdot 6$ & $30 \cdot 3$ & $27 \cdot 6,33 \cdot 0$ & $<0.001$ & $26 \cdot 7 \dagger$ & $24 \cdot 0,29 \cdot 5$ & $29 \cdot 8$ & $27 \cdot 1,32 \cdot 4$ & $<0.001$ \\
\hline Non-milk extrinsic sugars & $7 \cdot 7 \dagger$ & $4.7,10.7$ & 8.7 & $5 \cdot 8,11 \cdot 7$ & 0.001 & $7.5 \dagger$ & $4 \cdot 4,10 \cdot 5$ & $9 \cdot 0$ & $6 \cdot 1,11.9$ & $<0.001$ \\
\hline Intrinsic milk sugars and starch & $37.8 \dagger$ & $34.8,40.9$ & $38 \cdot 8$ & $35 \cdot 8,41.9$ & 0.001 & $34.3 \dagger$ & $32 \cdot 5,36 \cdot 2$ & 35.9 & $34 \cdot 4,37 \cdot 6$ & 0.003 \\
\hline Intrinsic milk sugarsł & $10 \cdot 0 \dagger$ & $8 \cdot 2,11 \cdot 8$ & 8.5 & $6 \cdot 8,10 \cdot 3$ & $<0.001$ & $9.9 \dagger$ & $8 \cdot 1,12 \cdot 1$ & $7 \cdot 9$ & $6 \cdot 5,9 \cdot 6$ & $<0.001$ \\
\hline $\begin{array}{l}\text { Non-starch polysaccharides (Englyst } \\
\text { Fibre, g) }\end{array}$ & $15 \cdot 1 \dagger$ & $13 \cdot 2,16 \cdot 9$ & $13 \cdot 3$ & $11 \cdot 4,15 \cdot 1$ & $<0.001$ & $15 \cdot 8 \dagger$ & $13 \cdot 9,17 \cdot 7$ & $13 \cdot 7$ & $11 \cdot 9,15 \cdot 5$ & $<0.001$ \\
\hline \multirow[t]{5}{*}{ Alcohol $(\mathrm{g}) \neq \S$} & $12 \cdot 1$ & $8 \cdot 8,16 \cdot 5$ & $12 \cdot 9$ & $9 \cdot 6,17 \cdot 5$ & $0 \cdot 290$ & $10 \cdot 0 \dagger$ & $7 \cdot 1,14 \cdot 0$ & $12 \cdot 7$ & $9 \cdot 6,16 \cdot 9$ & 0.013 \\
\hline & \multicolumn{4}{|c|}{ TNS-A } & & \multicolumn{4}{|c|}{ TNS-B } & \\
\hline & \multicolumn{2}{|c|}{ Consumers } & \multicolumn{2}{|c|}{ Non-consumers } & & \multicolumn{2}{|c|}{ Consumers } & \multicolumn{2}{|c|}{ Non-consumers } & \\
\hline & \multicolumn{2}{|r|}{$n 484$} & \multicolumn{2}{|c|}{$n 4254$} & & \multicolumn{2}{|c|}{$n 224$} & \multicolumn{2}{|c|}{$n 4514$} & \\
\hline & $\begin{array}{c}\text { Estimated } \\
\text { marginal } \\
\text { mean }\end{array}$ & $95 \% \mathrm{Cl}$ & $\begin{array}{c}\text { Estimated } \\
\text { marginal } \\
\text { mean }\end{array}$ & $95 \% \mathrm{Cl}$ & $P$-value & $\begin{array}{c}\text { Estimated } \\
\text { marginal } \\
\text { mean }\end{array}$ & $95 \% \mathrm{Cl}$ & $\begin{array}{c}\text { Estimated } \\
\text { marginal } \\
\text { mean }\end{array}$ & $95 \% \mathrm{Cl}$ & $P$-value \\
\hline \multicolumn{11}{|l|}{ Micronutrients $^{\star \star}$} \\
\hline Vitamin A (retinol equivalents) $(\mu \mathrm{g}) \mp \S$ & $904 \cdot 9 \dagger$ & $753.4,1086 . .9$ & 828.9 & $696 \cdot 5,986.5$ & 0.024 & $966 \cdot 1$ & $794 \cdot 0,1175 \cdot 2$ & 885.5 & $750 \cdot 9,1044 \cdot 2$ & 0.128 \\
\hline Vitamin D $(\mu \mathrm{g}) \neq \S$ & 2.5 & $2 \cdot 1,3 \cdot 0$ & $2 \cdot 4$ & $2 \cdot 0,2 \cdot 8$ & 0.213 & 2.4 & $2 \cdot 0,2 \cdot 9$ & 2.4 & $2 \cdot 0,2 \cdot 8$ & 0.912 \\
\hline Vitamin E (mg) $\ddagger$ & $11.6 \dagger$ & $10 \cdot 1,13 \cdot 0$ & $9 \cdot 8$ & $8 \cdot 4,11 \cdot 2$ & $<0.001$ & $11.6 \dagger$ & $10 \cdot 6,12 \cdot 7$ & $9 \cdot 3$ & $8 \cdot 6,10 \cdot 1$ & $<0.001$ \\
\hline Thiamin $(\mathrm{mg})$ & $1.4 \dagger$ & $1 \cdot 2,1.6$ & 1.3 & $1 \cdot 1,1.5$ & 0.001 & $1.5 \dagger$ & $1 \cdot 3,1 \cdot 7$ & 1.3 & $1 \cdot 1,1 \cdot 5$ & $<0.001$ \\
\hline Riboflavin (mg) & 1.5 & $1 \cdot 2,1 \cdot 7$ & 1.4 & $1 \cdot 2,1 \cdot 7$ & 0.196 & $1.5 \dagger$ & $1 \cdot 3,1 \cdot 8$ & 1.4 & $1 \cdot 2,1 \cdot 7$ & 0.003 \\
\hline Niacin equivalent (mg) & 33.5 & $28 \cdot 7,38 \cdot 3$ & 33.8 & $29 \cdot 1,38 \cdot 6$ & 0.486 & 34.4 & $29 \cdot 5,39 \cdot 3$ & 33.8 & $29 \cdot 1,38 \cdot 5$ & 0.382 \\
\hline Vitamin $\mathrm{B}_{6}(\mathrm{mg}) \ddagger$ & 1.9 & $1 \cdot 5,2 \cdot 3$ & 1.9 & $1 \cdot 5,2 \cdot 3$ & 0.992 & 1.9 & $1 \cdot 7,2 \cdot 1$ & 1.9 & $1 \cdot 8,2 \cdot 1$ & 0.530 \\
\hline Vitamin $B_{12}(\mu \mathrm{g}) \ddagger \S$ & $4 \cdot 6$ & $3 \cdot 6,6 \cdot 0$ & $4 \cdot 7$ & $3 \cdot 7,6 \cdot 0$ & 0.777 & 4.5 & $3 \cdot 9,5 \cdot 2$ & 4.8 & $4 \cdot 3,5 \cdot 5$ & 0.154 \\
\hline
\end{tabular}




\begin{tabular}{|c|c|c|c|c|c|c|c|c|c|c|}
\hline \multirow[b]{4}{*}{ Nutrients } & \multicolumn{4}{|c|}{ TNS-A } & \multirow[b]{4}{*}{$P$-value } & \multicolumn{4}{|c|}{ TNS-B } & \multirow[b]{4}{*}{$P$-value } \\
\hline & \multirow{2}{*}{\multicolumn{2}{|c|}{$\begin{array}{c}\text { Consumers } \\
n 484 \\
\end{array}$}} & \multirow{2}{*}{\multicolumn{2}{|c|}{$\frac{\text { Non-consumers }}{n 4254}$}} & & \multirow{2}{*}{\multicolumn{2}{|c|}{$\begin{array}{c}\text { Consumers } \\
n 224 \\
\end{array}$}} & \multirow{2}{*}{\multicolumn{2}{|c|}{$\begin{array}{c}\text { Non-consumers } \\
n 4514 \\
\end{array}$}} & \\
\hline & & & & & & & & & & \\
\hline & $\begin{array}{c}\text { Estimated } \\
\text { marginal } \\
\text { mean }\end{array}$ & $95 \% \mathrm{Cl}$ & $\begin{array}{c}\text { Estimated } \\
\text { marginal } \\
\text { mean }\end{array}$ & $95 \% \mathrm{Cl}$ & & $\begin{array}{c}\text { Estimated } \\
\text { marginal } \\
\text { mean }\end{array}$ & $95 \% \mathrm{Cl}$ & $\begin{array}{c}\text { Estimated } \\
\text { marginal } \\
\text { mean }\end{array}$ & $95 \% \mathrm{Cl}$ & \\
\hline Folate $(\mu \mathrm{g})$ & $242 \cdot 3 \dagger$ & $201 \cdot 9,282 \cdot 6$ & $229 \cdot 4$ & $189 \cdot 5,269 \cdot 2$ & 0.001 & $255 \cdot 7 \dagger$ & $214 \cdot 7,296 \cdot 6$ & 237.0 & $197 \cdot 5,276 \cdot 6$ & 0.001 \\
\hline Pantothenic acid (mg) & 5.6 & $4.6,6.5$ & $5 \cdot 3$ & $4.4,6 \cdot 3$ & 0.007 & $5.9 \dagger$ & $4.9,6.8$ & $5 \cdot 3$ & $4.4,6 \cdot 2$ & $<0.001$ \\
\hline Biotin $(\mu \mathrm{g})$ & $36.4 \dagger$ & $30 \cdot 1,42 \cdot 7$ & $30 \cdot 0$ & $23 \cdot 8,36 \cdot 3$ & $<0.001$ & $41.3 \dagger$ & $34 \cdot 9,47 \cdot 6$ & 31.7 & $25.5,37.9$ & $<0.001$ \\
\hline Vitamin C $(\mathrm{mg}) \ddagger \S$ & $79.1 \dagger$ & $58 \cdot 4,107 \cdot 3$ & $62 \cdot 1$ & $46 \cdot 0,83 \cdot 8$ & $<0.001$ & $90.4 \dagger$ & $75 \cdot 3,108 \cdot 6$ & $79 \cdot 0$ & $67 \cdot 8,92 \cdot 3$ & 0.012 \\
\hline Sodium $(\mathrm{mg})$ & $1854 \cdot 8 \dagger$ & $1596 \cdot 0,2113 \cdot 6$ & $2053 \cdot 9$ & $1798 \cdot 5,2309 \cdot 3$ & $<0.001$ & $1732 \cdot 2 \dagger$ & $1469 \cdot 6,1994.8$ & $2006 \cdot 2$ & $1752 \cdot 6,2259 \cdot 8$ & $<0.001$ \\
\hline Potassium (mg) & $2866.7 \dagger$ & $2595 \cdot 1,3138 \cdot 2$ & $2645 \cdot 3$ & $2377 \cdot 3,2913 \cdot 3$ & $<0.001$ & $3021.3+$ & $2746 \cdot 0,3296 \cdot 6$ & 2694.8 & $2428 \cdot 8,2960.5$ & $<0.001$ \\
\hline Calcium (mg) & $696 \cdot 6$ & $591 \cdot 3,801 \cdot 8$ & 702.8 & $599 \cdot 0,806 \cdot 7$ & 0.541 & $717 \cdot 4$ & $610 \cdot 8,824 \cdot 0$ & $709 \cdot 6$ & $606 \cdot 7,812 \cdot 6$ & 0.599 \\
\hline Magnesium (mg) & $276 \cdot 9 \dagger$ & $251 \cdot 0,302 \cdot 8$ & 237.8 & $212 \cdot 3,263 \cdot 4$ & $<0.001$ & $301.5 \dagger$ & $275 \cdot 3,327 \cdot 8$ & $245 \cdot 3$ & $219 \cdot 9,270 \cdot 6$ & $<0.001$ \\
\hline Phosphorus (mg) & $1165.8 \dagger$ & $1056 \cdot 1,1275 \cdot 6$ & 1125.4 & $1017 \cdot 1,1233 \cdot 7$ & $<0.001$ & $1191.5 \dagger$ & $1080 \cdot 3,1302 \cdot 6$ & $1126 \cdot 2$ & $1018 \cdot 9,1233.5$ & $<0.001$ \\
\hline Iron (mg) & $11.1 \dagger$ & $9.9,12.4$ & 10.4 & $9.1,11.6$ & $<0.001$ & $11.2 \dagger$ & $9 \cdot 9,12 \cdot 4$ & 10.5 & $9 \cdot 3,11 \cdot 7$ & $<0.001$ \\
\hline Copper (mg)‡§ & $1.2 \dagger$ & $1.0,1.4$ & 1.0 & $0.9,1.2$ & $<0.001$ & $1.4 \dagger$ & $1 \cdot 3,1.6$ & 1.2 & $1 \cdot 1,1 \cdot 3$ & $<0.001$ \\
\hline Zinc (mg) & $9.0 \dagger$ & $7 \cdot 9,10 \cdot 0$ & 8.8 & $7 \cdot 7,9 \cdot 8$ & 0.044 & $9.3 \dagger$ & $8 \cdot 2,10 \cdot 3$ & 8.8 & $7 \cdot 8,9 \cdot 8$ & 0.002 \\
\hline Chloride (mg) & $3017.1 \dagger$ & $2631 \cdot 3,3402 \cdot 9$ & $3286 \cdot 7$ & $2906 \cdot 0,3667.5$ & $<0.001$ & $2877.8 \dagger$ & $2486 \cdot 4,3269 \cdot 1$ & $3242 \cdot 2$ & $2864 \cdot 3,3620 \cdot 1$ & $<0.001$ \\
\hline Manganese (mg) & $3.3 \dagger$ & $2 \cdot 8,3 \cdot 8$ & $2 \cdot 8$ & $2 \cdot 3,3 \cdot 3$ & $<0.001$ & $3.5 \dagger$ & $3.0,4.0$ & 2.9 & $2.4,3.4$ & $<0.001$ \\
\hline lodine $(\mu \mathrm{g})$ & 161.9 & $131 \cdot 1,192 \cdot 7$ & 158.7 & $128 \cdot 3,189 \cdot 1$ & 0.282 & $158 \cdot 2$ & $127 \cdot 0,189 \cdot 4$ & $158 \cdot 6$ & $128 \cdot 5,188 \cdot 8$ & 0.914 \\
\hline Selenium $(\mu \mathrm{g}) \ddagger$ & $56.9 \dagger$ & $48 \cdot 3,65 \cdot 4$ & 51.9 & $43.4,60 \cdot 3$ & $<0.001$ & $54.9 \dagger$ & $49.8,60.5$ & 50.5 & $46 \cdot 6,54 \cdot 8$ & 0.004 \\
\hline
\end{tabular}

The actual sample size in the computation for vitamin A and vitamin D, for TNS-A consumers was 314 and for TNS-A non-consumers was 2172 , whereas for TNS-B consumers was 138 and for TNS-B non-consumers was 2348 . The actual

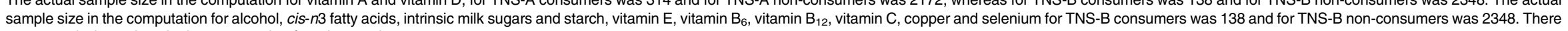
sample size in the computation for alcohol, cis-n3 fatty acids, intrinsic milk sug morsing values in the computation for other nutrients as outcomes.
were no

* *urvey-adjusted generalised linear model (GLM) with a linear link function and predictors such as age, sex, ethnicity, region of residency, socio-economic and smoking status was used for energy intake as an outcome for TNS-A; surveyadjusted GLM with a linear link function and predictors such as age, sex, ethnicity, region of residency, socio-economic and smoking status, alcohol and energy intakes was used for other macronutrient intake outcomes for TNS-A; surveyadjusted GLM with a linear link function and predictors such as age, sex, ethnicity, region of residency, socio-economic and smoking status, and energy intake was used for alcohol intake as an outcome for TNS-A. The same statistical analysis

was conducted for TNS-B, but region of residency was excluded from predictors.

₹Geometric marginal means were presented due to non-normally distributed residual data in TNS-B population.

SGeometric marginal means were presented due to non-normally distributed residual data in TNS-A population.

${ }^{*}$ Survey-adjusted GLM with a linear link function and predictors: age, sex, ethnicity, region of residency, socio-economic and smoking status, alcohol and energy intakes was used for TNS-A. The same statistical analysis was conducted for TNS-B but region of residency was excluded from predictors. 


\section{CVD risk markers}

Blood samples were not available from all participants, and anthropometric and blood pressure data were also missing. Associations between TNS consumption and CVD risk markers were analysed for the remaining participants. The estimated marginal mean $(95 \% \mathrm{CI})$ values of CVD risk markers are shown in Table 3. For TNS-A consumers, BMI, WC, SBP and DBP were significantly lower, and HDL was significantly higher compared to non-consumers. For those consuming $>7.08 \mathrm{~g}$ TNS/d (TNS-B), only WC, SBP and DBP were significantly lower compared to non-consumers (data shown in online supplementary material). Surveyadjusted regression analysis showed that for every gram increase in TNS consumption per $4184 \mathrm{~kJ}$ of adults' energy intake (Table 3), SBP was significantly lower demonstrating a dose-response relationship $(P=0.028)$.

\section{Discussion}

Interventional and observational evidence suggests that replacing refined carbohydrate-based snacks with tree nut snacks may improve blood lipid profiles, management of body weight ${ }^{(33,34)}$ and nutrient intakes. However, TNS intakes in the general UK population have not been fully investigated. Previous studies have been conducted in the NHANES US adult population ${ }^{(20,21)}$ using multiple 24-h dietary recalls to collect food intake data. This crosssectional analysis using a representative UK adult population revealed that just $10 \%$ of respondents reported consuming any amount of TNS during their 4-d food intake recording period, just less than $5 \%$ reported consuming more than $7 \cdot 08 \mathrm{~g}(1 / 4 \mathrm{Oz})$ per day on average (around a handful over the $4-\mathrm{d}$ period), and only $0.34 \%$ reported consuming the US Food and Drug Administration recommendation of $42.5 \mathrm{~g}$ per day ${ }^{(35)}$. The relatively small sub-population of TNS consumers was more likely to be female, white, older and living in England and less likely to be current smokers relative to non-consumers.

Increments in TNS consumption (g per $4184 \mathrm{~kJ}$ of energy intake) were not associated with significantly greater modified MDS and HDS in consumers. This lack of doseresponse relationship could be due to the low consumption of TNS in the population (for TNS-A consumers, median $0.8 \%$ of total energy intake and $6.5 \mathrm{~g} / \mathrm{d}$ in terms of total weight intake; for TNS-B consumers, $2 \cdot 3 \%$ of total energy intake, and $14.0 \mathrm{~g} / \mathrm{d}$ in terms of total weight intake). TNS consumption status may be an indicator of improved overall diet quality, but the actual amount consumed has very little practical impact.

Since TNS consumption status appears to act as a marker of healthy dietary patterns, it is not surprising that the overall nutrient intake profile of TNS consumers was more favourable compared to non-consumers. The contribution of non-milk extrinsic sugar intakes to energy was only marginally lower in TNS consumers (a difference of

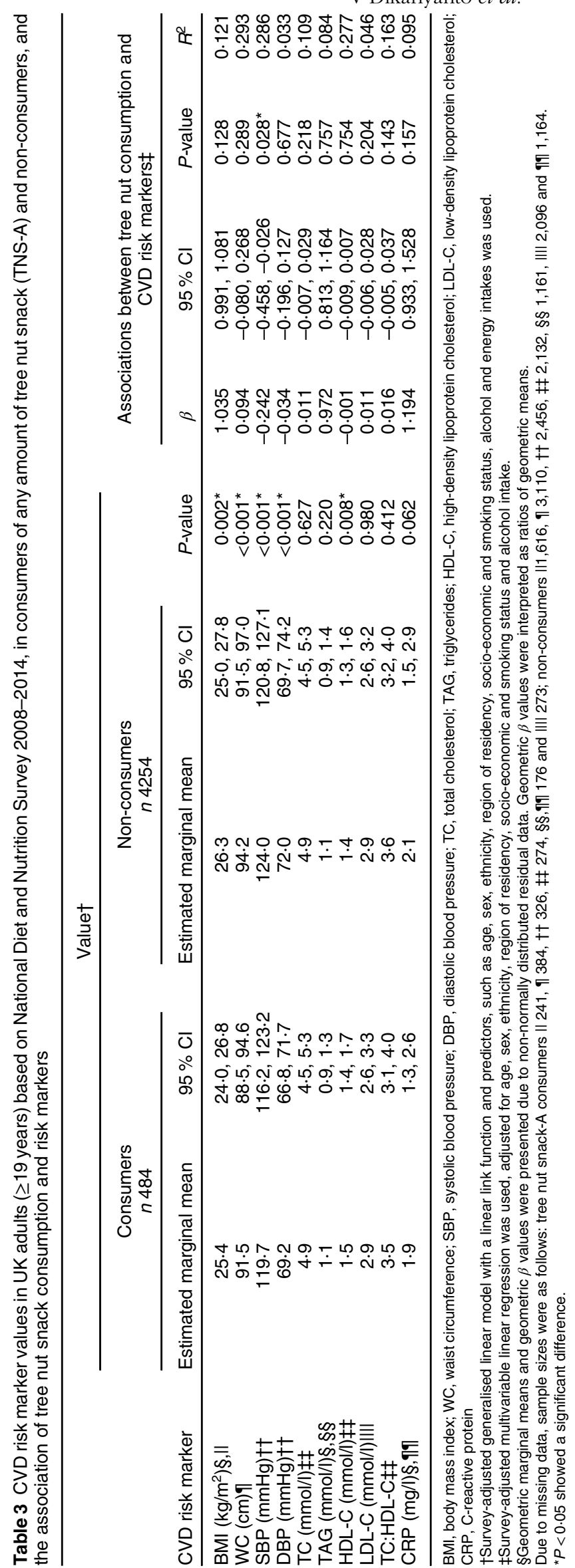


$1.0 \%$ of energy). Fibre (non-starch polysaccharides) intakes were $1.8 \mathrm{~g}$ higher in TNS consumers compared with non-consumers, but TNS intake is unlikely to contribute more than a third of this difference, with the remainder due to greater intakes of other fibre-rich foods.

The observations reported here and in the US population imply that TNS are usually eaten as a part of an overall healthier dietary pattern in industrialised countries ${ }^{(21)}$, which would be predicted to translate to better cardiovascular health outcomes. UK TNS consumers had significantly reduced BMI, WC, SBP and DBP and significantly higher HDL-C, but the slightly lower mean CRP in TNS consumers did not reach statistical significance compared with non-consumers. O'Neil et al.(2015) previously reported that $\geq 7.08 \mathrm{~g}$ tree nut consumption was associated with lower BMI and WC, as well as SBP and higher HDL-C, in the US adult population adjusted for the same covariates as used in the present analysis, plus physical activity level ${ }^{(20)}$. Overall feeding trials have reported that higher tree nut consumption did not result in weight gain ${ }^{(18,19)}$, which may be related to their satiating/satiety-inducing properties $^{(36)}$, as well as limited lipid bioaccessibility ${ }^{(37)}$. Since TNS consumers' median intake was low in the UK, observed differences in BMI and WC could be related to confounding factors such as physical activity levels, which was not considered in the present analysis due to lack of available data. Mean SBP was $4.3 \mathrm{mmHg}$ lower and mean DBP was $2.8 \mathrm{mmHg}$ lower in TNS consumers compared to non-consumers, a clinically meaningful difference that would be predicted to reduce risk of CVD. The SUN prospective cohort study reported that there was no association between tree nut consumption and blood pressure; the potential reasons could be an underestimated amount of nut consumption, no assessment on the change in nut consumption during follow-up, and no specific information on preparation method, for example, salted, roasted or raw ${ }^{(38)}$. The Physician's Health Study observed blood pressure reduction only in lean volunteers ${ }^{(39)}$. A recent metaanalysis of 21 randomised control trials reported that total nut consumption lowered SBP in participants without type 2 diabetes, and mixed nuts also lowered $\mathrm{DBP}^{(40)}$. Although plasma CRP concentrations were not significantly different in the NDNS cohort, a cross-sectional study using data from the Nurse's Health Study and Health Professional FollowUp Study revealed that consumers eating tree nuts $\geq 5$ times weekly based on FFQ had significantly lower CRP ${ }^{(41)}$, suggesting larger differences in intake may be required to impact on systemic inflammatory markers. However, a meta-analysis of 20 randomised controlled trials suggested that tree nut consumption did not reduce $\mathrm{CRP}^{(42)}$. In the current study, the amount of nuts consumed by consumers in the current UK cohort was low, and therefore, the SBP and DBP differences observed are likely to be the sum effect of an overall healthier dietary pattern including $\mathrm{TNS}^{(43-46)}$.

A significant difference was observed in HDL-C between TNS consumers and non-consumers. Cross-sectional analysis in the US adult population also reported higher HDL-C in TNS consumers ${ }^{(20)}$. There were no significant differences observed in other blood lipids. A recent meta-analysis of 61 interventional clinical trials revealed that tree nut intake reduced TC, TAG and LDL-C, and it was reported that the dosage of tree nut intake determined cholesterol lowering capacity rather than the nut types ${ }^{(47)}$. A pooled analysis of 25 feeding trials conducted in seven countries demonstrated the reduction of TC, LDL-C and the ratio of TC to HDL-C but failed to report the increase of HDL-C in response to tree nut intake ${ }^{(48)}$. These inconsistent associations of tree nut consumption and blood pressure, CRP and blood lipids between cross-sectional analysis and clinical trials could be due to different dosage and duration of consumption (duration of the study), residual confounding effects, characteristics such as baseline lipid profile, as well as study sample size relating to statistical power ${ }^{(41)}$.

Strengths of the current study include using a relatively large, nationally representative UK population, and the close agreement with results reported in a nationally representative US population suggests that findings may be generalisable to other industrialised countries with similar dietary profiles. The availability of estimated portion size food diary data over a 4-d period is considered to be one of the more accurate dietary assessment methods in large populations, although underreporting of energy intake is a well-known problem with this methodology that limits the conclusions that can be drawn. Furthermore, the use of 4-d estimated food diaries means that significant nut intakes on other days may have been missed and a significant proportion of TNS consumers may have been wrongly classified as non-consumers; analysis based on frequency of tree nut consumption was not possible. Available information on physical activity was incomplete so statistical analysis models could not be adjusted for this potentially confounding factor. Different types of tree nuts have differing nutrient profiles and potentially nutrient bioaccessibility, and therefore it may be misleading to group them altogether in terms of associations with CVD risk factors. In addition to that, missing data for CVD risk factors resulted in lower sample sizes.

In conclusion, the prevalence of TNS consumers in the UK adult population is estimated to be approximately $10 \%$, and median intakes were low in the group classified as TNS consumers. Tree nut snack consumption was associated with higher diet quality scores and a more favourable nutrient intake profile. Tree nut snack consumption may be a marker of a healthy dietary pattern and is associated with lower adiposity and blood pressure. It is recommended that tree nuts should replace high refined carbohydrate-based snacks as part of a healthy diet. To determine the relative contribution of tree nuts to the sum impact of a healthier dietary pattern on risk of CVD, future randomised controlled trials should investigate the effect of replacing usual refined carbohydrate snacks with tree nuts on markers of cardiometabolic disease risk. 


\section{Acknowledgements}

Acknowledgements: We thank the two anonymous reviewers for their valuable insights. Financial support: This work was supported in part by the Almond Board of California (grant number 15-BERRYS-01) and the Indonesia Endowment Fund for Education. The funders had no role in the design, analysis or writing of this article. Conflict of interest: None. Authorship: The authors' responsibilities were as follows: V.D., W.L.H., S.E.B. and G.K.P. designed the research; V.D. analysed the data; L.F. advised on analysis of the data; V.D. and W.L.H. wrote the manuscript; W.L.H. had primary responsibility for final content. All authors read, commented and approved the final manuscript. Ethics of human subject participation: The current study was conducted according to the guidelines laid down in the Declaration of Helsinki, and all procedures involving human subjects were approved by the National Health Service Local Research Ethics Committee covering each of the postal sectors. Written informed consent was obtained from all participants.

\section{Supplementary material}

For supplementary material accompanying this paper visit https://doi.org/10.1017/S1368980019003914

\section{References}

1. Bord Bia - The Irish Food Board (2018) Healthy snacking UK and Ireland. https://www.bordbia.ie/globalassets/bordbia. ie/industry/marketing-reports/consumer-reports/healthysnacking-uk-and-ireland-january-2018.pdf (accessed June 2019).

2. International Nut \& Dried Fruit Council (2016) Nuts \& dried fruits: Statistical yearbook 2016/2017. https://www.nutfruit. org/consumers/news/detail/statistical-yearbook-2016-17 (accessed September 2017).

3. U.S. Department of Agriculture. Agricuture Research Service. Food Data Central (2019) Nuts, almonds. https://fdc.nal.usda. gov/fdc-app.html\#/food-details/170567/nutrients (accessed June 2019).

4. Chen CY \& Blumberg JB (2008) Phytochemical composition of nuts. Asia Pac J Clin Nutr 17, Suppl 1, 329-332.

5. Maguire LS, O'Sullivan SM, Galvin K et al. (2004) Fatty acid profile, tocopherol, squalene and phytosterol content of walnuts, almonds, peanuts, hazelnuts and the macadamia nut. Int J Food Sci Nutr 55, 171-178.

6. Rajaram S, Haddad EH, Mejia A et al. (2009) Walnuts and fatty fish influence different serum lipid fractions in normal to mildly hyperlipidemic individuals: a randomized controlled study. Am J Clin Nutr 89, 1657s-1663s.

7. Torabian S, Haddad E, Cordero-MacIntyre Z et al. (2010) Long-term walnut supplementation without dietary advice induces favorable serum lipid changes in free-living individuals. Eur J Clin Nutr 64, 274-279.

8. Tapsell LC, Batterham MJ, Teuss G et al. (2009) Long-term effects of increased dietary polyunsaturated fat from walnuts on metabolic parameters in type II diabetes. Eur J Clin Nutr 63, 1008-1015.
9. Jenkins DJ, Kendall CW, Marchie A et al. (2008) Almonds reduce biomarkers of lipid peroxidation in older hyperlipidemic subjects. J Nutr 138, 908-913.

10. Gebauer SK, West SG, Kay CD et al. (2008) Effects of pistachios on cardiovascular disease risk factors and potential mechanisms of action: a dose-response study. Am J Clin Nutr 88, 651-659.

11. Griel AE, Cao Y, Bagshaw DD et al. (2008) A macadamia nut-rich diet reduces total and LDL-cholesterol in mildly hypercholesterolemic men and women.J Nutr 138, 761-767.

12. Mercanligil SM, Arslan P, Alasalvar C et al. (2007) Effects of hazelnut-enriched diet on plasma cholesterol and lipoprotein profiles in hypercholesterolemic adult men. Eur J Clin Nutr 61, 212-220.

13. Mukuddem-Petersen J, Stonehouse-Oosthuizen W, Jerling JC et al. (2007) Effects of a high walnut and high cashew nut diet on selected markers of the metabolic syndrome: a controlled feeding trial. Br J Nutr 97, 1144-1153.

14. Casas-Agustench P, Lopez-Uriarte P, Bullo M et al. (2011) Effects of one serving of mixed nuts on serum lipids, insulin resistance and inflammatory markers in patients with the metabolic syndrome. Nutr Metab Cardiovasc Dis 21, 126-135.

15. Ros E (2010) Health benefits of nut consumption. Nutrients $\mathbf{2}$, 652-682.

16. Jimenez-Gomez Y, Lopez-Miranda J, Blanco-Colio LM et al. (2009) Olive oil and walnut breakfasts reduce the postprandial inflammatory response in mononuclear cells compared with a butter breakfast in healthy men. Atherosclerosis $\mathbf{2 0 4}$, e70-e76.

17. Ros E, Nunez I, Perez-Heras A et al. (2004) A walnut diet improves endothelial function in hypercholesterolemic subjects: A randomized crossover trial. Circulation 109, 1609-1614.

18. Flores-Mateo G, Rojas-Rueda D, Basora J et al. (2013) Nut intake and adiposity: Meta-analysis of clinical trials. Am J Clin Nutr 97, 1346-1355.

19. Wien MA, Sabate JM, Ikle DN et al. (2003) Almonds vs complex carbohydrates in a weight reduction program. Int JObes Relat Metab Disord 27, 1365-1372.

20. O'Neil CE, Fulgoni VL 3rd \& Nicklas TA (2015) Tree Nut consumption is associated with better adiposity measures and cardiovascular and metabolic syndrome health risk factors in U.S. Adults: NHANES 2005-2010. Nutr J 14, 64.

21. O'Neil CE, Nicklas TA \& Fulgoni VL 3rd (2015) Tree nut consumption is associated with better nutrient adequacy and diet quality in adults: National Health and Nutrition Examination Survey 2005-2010. Nutrients 7, 595-607.

22. Bates B, Lennox A, Prentice A et al. (2014) National Diet and Nutrition Survey Results from Years 1, 23 and 4 (combined) of the Rolling Programme (2008/2009-2011/2012). London: Public Health England.

23. Bates B, Cox L, Nicholson S et al. (2016) National Diet and Nutrition Survey Results from Years 5 and 6 (combined) of the Rolling Programme (2012/2013-2013/2014). London: Public Health England.

24. Couto E, Boffetta P, Lagiou P et al. (2011) Mediterranean dietary pattern and cancer risk in the EPIC cohort. $\mathrm{Br} J$ Cancer 104, 1493-1499.

25. Maynard M, Ness AR, Abraham L et al. (2005) Selecting a healthy diet score: Lessons from a study of diet and health in early old age (the Boyd Orr cohort). Public Health Nutr 8, 321-326.

26. Nutrition Science Team. Public Health England (2016) Government Recommendations for Energy and Nutrients for Males and Females Aged 1-18 years and 19+ years. London: Department of Health.

27. DH Cardiovascular Disease Team (2013) Cardiovascular Disease Outcomes Strategy: Improving Outcomes for People with or at Risk of Cardiovascular Disease. London: Department of Health. 
28. Public Health England (2016) The Eatwell Guide. London, UK: Department of Health.

29. Scientific Advisory Committee on Nutrition (2018) Draft Report: Saturated Fats and Health. London: Department of Health.

30. Scientific Advisory Committee on Nutrition (2015) Carbohydrates and Health. London: The Stationery Office.

31. NatCen Social Research, The Medical Research Council Elsie Widdowson Laboratory (MRC EWL) \& University College London (UCL) (2008) National Diet and Nutrition Survey Years 1-4 2008/09-2011/12: User Guide for UK Data (core $\varepsilon$ country boost data). London: Public Health England.

32. NatCen Social Research \& The Medical Research Council Elsie Widdowson Laboratory (MRC EWL) (2012) National Diet and Nutrition Survey Years 5-6 2012/13-2013/14. User Guide for UK Data. London: Public Health England.

33. Li Z, Song R, Nguyen C et al. (2010) Pistachio nuts reduce triglycerides and body weight by comparison to refined carbohydrate snack in obese subjects on a 12-week weight loss program. J Am Coll Nutr 29, 198-203.

34. Rehm CD \& Drewnowski A (2017) Replacing American snacks with tree nuts increases consumption of key nutrients among US children and adults: Results of an NHANES modeling study. Nutr J 16, 17.

35. U. S. Food \& Drug Administration (2003) Qualified health claims: Letter of enforcement discretion - nuts and coronary heart disease (Docket No 02P-0505). http://wayback. archive-it.org/7993/20171114183724/https://www.fda.gov/ Food/IngredientsPackagingLabeling/LabelingNutrition/ucm 072926.htm (accessed September 2017).

36. Mattes RD \& Dreher ML (2010) Nuts and healthy body weight maintenance mechanisms. Asia Pac J Clin Nutr 19, 137-141.

37. Mandalari G, Grundy MM, Grassby T et al. (2014) The effects of processing and mastication on almond lipid bioaccessibility using novel methods of in vitro digestion modelling and micro-structural analysis. Br J Nutr 112, 1521-1529.

38. Martinez-Lapiscina EH, Pimenta AM, Beunza JJ et al. (2010) Nut consumption and incidence of hypertension: the SUN prospective cohort. Nutr Metab Cardiovasc Dis 20, 359-365.

39. Djousse L, Rudich T \& Gaziano JM (2009) Nut consumption and risk of hypertension in US male physicians. Clin Nutr $\mathbf{2 8}$, $10-14$.

40. Mohammadifard N, Salehi-Abargouei A, Salas-Salvado J et al. (2015) The effect of tree nut, peanut, and soy nut consumption on blood pressure: a systematic review and meta-analysis of randomized controlled clinical trials. Am J Clin Nutr 101, 966-982.

41. Yu Z, Malik VS, Keum N et al. (2016) Associations between nut consumption and inflammatory biomarkers. Am J Clin Nutr 104, 722-728.

42. Mazidi M, Rezaie P, Ferns GA et al. (2016) Impact of different types of tree nut, peanut, and soy nut consumption on serum C-reactive protein (CRP): a systematic review and metaanalysis of randomized controlled clinical trials. Medicine 95, e5165.

43. Kahleova H, Levin S \& Barnard N (2017) Cardio-metabolic benefits of plant-based diets. Nutrients $\mathbf{9}, 8$.

44. Lin PH, Allen JD, Li YJ et al. (2012) Blood pressure-lowering mechanisms of the DASH dietary pattern. J Nutr Metabol 2012, 472396.

45. Utsugi MT, Ohkubo T, Kikuya M et al. (2008) Fruit and vegetable consumption and the risk of hypertension determined by self measurement of blood pressure at home: the Ohasama study. Hypertens Res 31, 1435-1443.

46. Hoshi T, Wissuwa B, Tian Y et al. (2013) Omega-3 fatty acids lower blood pressure by directly activating large-conductance $\mathrm{Ca}(2)(+)$-dependent $\mathrm{K}(+)$ channels. Proc Natl Acad Sci USA 110, 4816-4821.

47. Del Gobbo LC, Falk MC, Feldman R et al. (2015) Effects of tree nuts on blood lipids, apolipoproteins, and blood pressure: systematic review, meta-analysis, and doseresponse of 61 controlled intervention trials. Am J Clin Nutr 102, $1347-1356$.

48. Sabate J, Oda K \& Ros E (2010) Nut consumption and blood lipid levels: a pooled analysis of 25 intervention trials. Arch Intern Med 170, 821-827. 